\title{
Precise estimation of shell model energy by second order extrapolation method
}

\author{
Takahiro Mizusaki \\ Institute of Natural Sciences, Senshu University, \\ Higashimita, Tama, Kawasaki, Kanagawa, 214-8580, Japan \\ Masatoshi Imada \\ Institute for Solid State Physics, University of Tokyo, Kashiwanoha, Kashiwa, 277-8581, Japan
}

(Dated: December 31, 2018)

\begin{abstract}
A second order extrapolation method is presented for shell model calculations, where shell model energies of truncated spaces are well described as a function of energy variance by quadratic curves and exact shell model energies can be obtained by the extrapolation. This new extrapolation can give more precise energy than those of first order extrapolation method. It is also clarified that first order extrapolation gives a lower limit of shell model energy. In addition to the energy, we derive the second order extrapolation formula for expectation values of other observables.
\end{abstract}

PACS numbers: 21.60.Cs

A pursuit for new numerical algorithms to solve nuclear shell model problems has been one of the most intriguing issues in nuclear structure physics. In the last decade, several new methods [1, 2, 3, [4, [5, 6, 7], 8] were proposed and have been developed. Consequently shell model calculation remarkably widens its capability beyond the limitation of conventional approaches such as Lanczos shell model diagonalization. Among these new methods, several extrapolation techniques [6, 7, 8] were proposed in order to reinforce a restricted power of Lanczos shell model calculations. We advocated the Lanczos shell model diagonalization with the help of the recently proposed non-empirical extrapolation technique [9, 10] for correlated electron systems and succeeded in enlarging the feasibility of large-scale shell model calculations by the extrapolation [8].

In general, interpolation can be reliable if the considered quantity behaves smoothly, while extrapolation cannot be guaranteed. A reliable extrapolation method needs a theory predicting how the considered quantity behaves in the extrapolated region. In the recently proposed extrapolation 9, 10], it has been shown that, exact energies defined in the whole space can be extrapolated from series of energies in truncated Hilbert spaces. If these truncated Hilbert spaces give fairly good approximations, energies of truncated spaces can be shown to behave linearly as a function of the energy variance. Therefore exact energies can be estimated by linearly extrapolated energies of truncated spaces into zero energy variance. In condensed matter physics there are good examples of this extrapolation in analyses of strongly correlated electrons on a lattice [11,12]. In Ref. [8] it has been shown that this extrapolation works also when applied to the nuclear shell model. However, for detailed spectroscopic studies, precise excitation energies are important, and this requires a more precise evaluation of the energy eigenvalues. Another problem is, as it will be discussed later, that the extrapolated shell model energies are systematically lower than the true energy eigenvalues. To solve this problem, in the present paper, we successfully improve the extrapolation method by including the second order term. Hereafter this extension is called second order extrapolation, while the previous one is called first order extrapolation.

In order to present our new method, we consider the relation between energy difference and modified energy variance as below. We define the difference $\delta E$ between the lowest energy eigenvalue $\langle\hat{H}\rangle$ in a given truncated space and true energy eigenvalue $\langle\hat{H}\rangle_{0}$ as

$$
\delta E=\langle\hat{H}\rangle-\langle\hat{H}\rangle_{0}
$$

The modified energy variance $\Delta E$ in the truncated space is also defined as

$$
\Delta E=\left\langle\hat{H}^{2}\right\rangle-\langle\hat{H}\rangle^{2} .
$$

Note that this definition of energy variance is different from the previous one [ 8$]$. Throughout the present paper, this new definition is used.

An approximate ground state $|\psi\rangle$ can be decomposed as the sum of the true eigenstate $\left|\psi_{0}\right\rangle$ and the rest component $\left|\psi_{r}\right\rangle$ as

$$
\begin{gathered}
|\psi\rangle=c\left|\psi_{0}\right\rangle+d\left|\psi_{r}\right\rangle, \\
\left|\psi_{r}\right\rangle=\sum_{n \neq 0} c_{n}\left|\psi_{n}\right\rangle,
\end{gathered}
$$

where $c, d$ and $c_{n}$ 's are coefficients, whose normalization condition is taken as $c^{2}+d^{2}=1$ and $\sum_{n \neq 0} c_{n}^{2}=1$. The $\left|\psi_{0}\right\rangle$ and $\left|\psi_{n}\right\rangle$ 's form an orthonormarized complete set spanning the whole space and its energy eigenvalue is denoted by $E_{n}$. By defining the moments $D_{j}(j=1,2, \ldots)$ as

$$
D_{j}=\sum_{n \neq 0} \frac{c_{n}^{2}\left(E_{n}-E_{0}\right)^{j}}{E_{0}^{j}},
$$


we can rewrite $\delta E$ and $\Delta E$ as

$$
\delta E=d^{2} D_{1} E_{0},
$$

and

$$
\Delta E=d^{2} D_{2} E_{0}^{2}-\left(d^{2}\right)^{2}\left(D_{1} E_{0}\right)^{2} .
$$

By eliminating $d^{2}$, we obtain

$$
\delta E=\frac{1}{2 A}\left[1-\sqrt{1-4 A^{2} \Delta E}\right]
$$

where

$$
A=\frac{D_{1}}{D_{2} E_{0}} .
$$

If $4 A^{2} \Delta E$ is small enough compared to the unity, we can expand $\delta E$ as

$$
\delta E=A \Delta E+A^{3} \Delta E^{2}+\ldots
$$

As the ratio of the moments $A$ embodies the structure of the rest component, $A$ can be a function of $d^{2}$ which is a small parameter. Here we assume that $A$ can be expanded by a function of $d^{2}$ as

$$
A=A_{0}+A_{1}^{\prime} d^{2}+O\left(d^{4}\right),
$$

where $A_{0}$ and $A_{1}^{\prime}$ are coefficients. This expansion is shown to be reasonable in the later discussion. By eliminating $d^{2}$ by Eq. (7), $A$ can be rewritten as

$$
A=A_{0}+A_{1} \Delta E+\ldots
$$

where $A_{1}$ is a new coefficient for $A_{1}^{\prime}$ with some additional factors. Therefore, we obtain the following second order extrapolation formula,

$$
\delta E=A_{0} \Delta E+\left(A_{0}^{3}+A_{1}\right) \Delta E^{2}+\ldots .
$$

The expansion parameter of Eqs. (12) and (13) is $\Delta E / E_{0}^{2}$ but, as $E_{0}$ is unknown, we present a new extrapolation formula by including the $E_{0}$ depedence in the expansion coefficients. We comment that, a dimensionless definition of energy variance as $\tilde{\Delta} E=\Delta E /\langle\hat{H}\rangle_{0}^{2}$ leads to the same result.

The first term of Eq. (13) shows the effect of the decrease of the norm of rest component. If we consider only the first term, this extrapolation is nothing but the previous first order extrapolation [8]. The second term shows the second order effects of the decrease of the norm of rest component $\left(A_{0}^{3}\right)$ and the first order effect of the structure change $\left(A_{1}\right)$. The sign of the second order coefficient will be clarified later in the discussion. This new method allows us to extrapolate shell model energies in the limit $\Delta E \rightarrow 0$ after determining the expansion coefficients in Eq. (13) by fitting to the shell model energies of various truncation spaces.
In order to investigate the properties of the present second order extrapolation method, we utilize numerically solvable shell model calculations. One frontier of the state-of-the-art large-scale shell model calculations is the $f p$ shell. Here we take ground state energies of ${ }^{48} \mathrm{Cr}$ and ${ }^{49} \mathrm{Cr}$ as $f p$ shell nuclei. For a numerical test, we consider two cases of different nature. The $M$-scheme dimensions are about 2 and 5 Millions for ${ }^{48,49} \mathrm{Cr}$, respectively, and we can easily diagonalize the Hamiltonian matrices by a shell model code, for instance, MSHELL [13]. As an effective shell model interaction, the KB3 interaction 14] is taken.

We consider two kinds of truncation scheme. The first is $\oplus_{s \leq t}\left(f_{7 / 2}\right)^{A-40-s}(r)^{s}$ where $r$ means the set of the $f_{5 / 2}, p_{3 / 2}$ and $p_{1 / 2}$ orbits and $t$ is the maximum number of particles allowed to be excited into the $r$ orbits. This truncation scheme is natural due to a considerable shell gap between the $f_{7 / 2}$ orbit and the others. We call this truncation scheme (I). The other is the truncation scheme (II): $\oplus_{s+s^{\prime} \leq t_{0}, s^{\prime} \leq t}\left(f_{7 / 2}\right)^{A-40-s-s^{\prime}}\left(p_{3 / 2}\right)^{s}\left(r^{\prime}\right)^{s^{\prime}}$ where $r^{\prime}$ means the set of the $f_{5 / 2}$ and $p_{1 / 2}$ orbits. Truncation spaces are specified by $t$ and $t_{0}$. For ${ }^{48,49} \mathrm{Cr}$, the $t_{0}$ is set to 5 , which means up to 5 nucleons in the $p_{3 / 2}$ and $r^{\prime}$ orbits. The parameter $t$ controls the size of the shell model space by specifying the maximum number of nucleons in the $r^{\prime}$ orbits. These two sets of truncation scheme gradually cover the whole shell model space in different ways as $t$ increases.
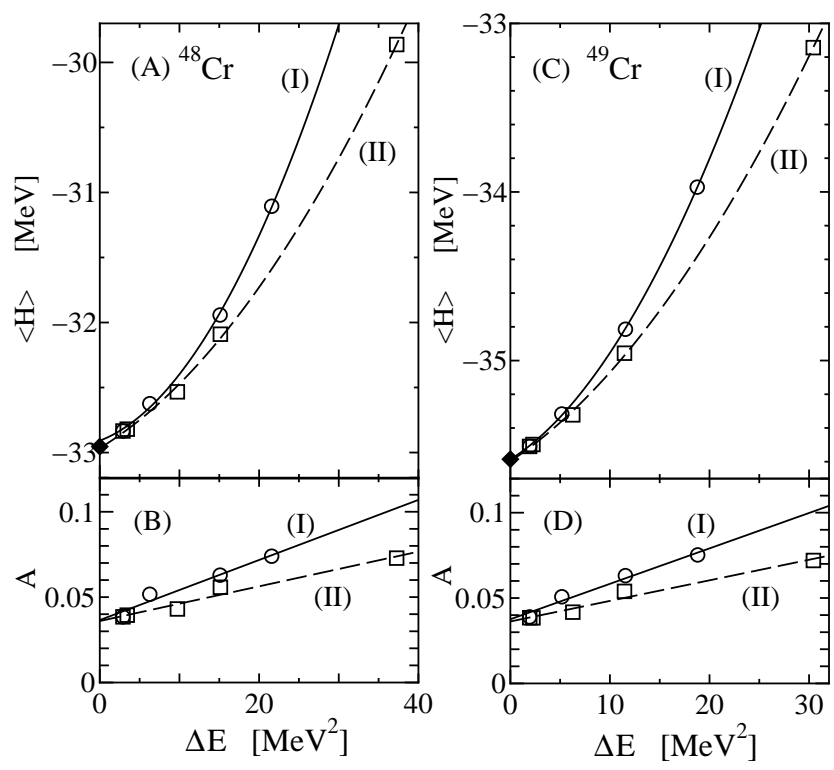

FIG. 1: Second order extrapolations of ground state energies into zero energy variance; (A) for ground state $\left(0_{1}^{+}\right)$of ${ }^{48} \mathrm{Cr}$, and $(\mathrm{C})$ for ground state $\left((5 / 2)_{1}^{-}\right)$of ${ }^{49} \mathrm{Cr}$. Open circles and open squares are energies for the truncated shell model spaces. Exact ground state energies are shown by filled diamonds. Results of two kinds of truncation scheme are shown for solid (I) and dashed (II) curves for (A) and (C). The ratio of the monents $A$ is shown as a function of $\Delta E$ for (B) and (D). 
In Figs. $1(\mathrm{~A})$ and $(\mathrm{C})$, the energies for the truncated shell model spaces with $t=2 \sim 5$ for the truncation scheme (I) and $t=1 \sim 5$ for the truncation scheme (II) are shown as a function of the energy variance $\Delta E$ for the ground states of ${ }^{48} \mathrm{Cr}$ and ${ }^{49} \mathrm{Cr}$. The solid (I) and dashed (II) curves show the second order extrapolations. Independently from the nature of the nuclei and from the adopted truncation scheme, the shell model energies for truncated spaces are well-fitted by quadratic curves as indicated by the second order extrapolation formula. These extrapolated energies are quite accurate, for examples, for ${ }^{48} \mathrm{Cr}$, they are -32.91 and $-32.97 \mathrm{MeV}$ for (I) and (II), respectively, while exact energy is $-32.95 \mathrm{MeV}$. For ${ }^{49} \mathrm{Cr}$, extrapolated energies are -35.59 and $-35.60 \mathrm{MeV}$ for (I) and (II), respectively, while exact one is -35.59 $\mathrm{MeV}$. Different truncation schemes can give almost the same extrapolation energy. This fact is quite useful for confirming the reliability of the extrapolated energy.

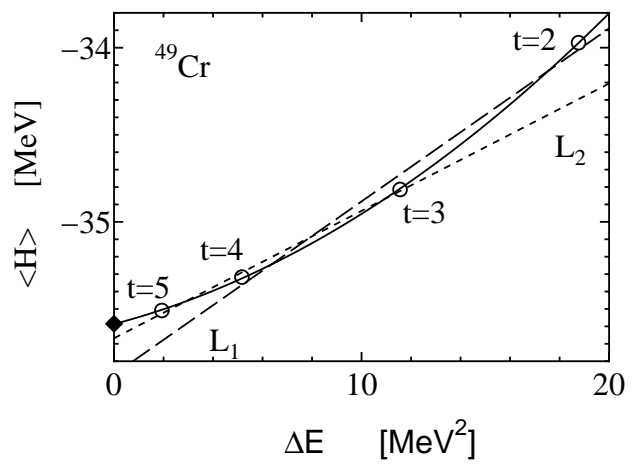

FIG. 2: First order extrapolations $\left(\mathrm{L}_{1}\right.$ and $\left.\mathrm{L}_{2}\right)$ and second order extrapolation (solid curve) are shown for the ground state of ${ }^{49} \mathrm{Cr}$.

In Fig.2, we compare the results of the first order extrapolation and of the second order extrapolation for the ground state of ${ }^{49} \mathrm{Cr}$, where the truncation scheme (I) is taken. $\mathrm{L}_{1}$ and $\mathrm{L}_{2}$ are the extrapolations from the shell model energies for $t=2,3,4$ and $t=3,4,5$, respectively. $\mathrm{L}_{2}$ gives higher extrapolation energy than $\mathrm{L}_{1}$, while it is still lower than the exact energy. However, globally the shell model energies for truncated spaces can be better fitted by a quadratic curve than by straight lines. The first order extrapolation is approximately parallel to the average tangential line of this quadratic curve around the fitted shell model energies. If the quadratic curve is upwardly concave, that is $A_{0}^{3}+A_{1}>0$, the extrapolated energy becomes a lower limit of shell model energy and approaches the exact energy from the lower side.

We further go into the details of the second order extrapolation formula. As we can obtain exact ground state wavefunctions for ${ }^{48,49} \mathrm{Cr}$, the moments $D_{1}$ and $D_{2}$ can also be evaluated. In Figs. 1 (B) and (D), the ratio of the moments $A$ is plotted as a function of $\Delta E$. These figures show that $A$ can be well described by the linear relation of $\Delta E$. The coefficient $A_{0}$ is almost the same for different truncation schemes, while $A_{1}$ is different. For
${ }^{48} \mathrm{Cr}$, the moment ratios are $A=0.037+0.0018 \Delta E$ for (I) and $A=0.036+0.0010 \Delta E$ for (II). In the second order coefficient, $A_{1}$ is positive for both cases. Moreover, $A_{1}$ is much larger than $A_{0}^{3}$. This means that it is important to take into account the structure change of the rest component in second order term.

As the moments $D_{1}$ and $D_{2}$ are positive by definition, the ratio of the moments $A$ is positive then $A_{0}$ is also positive. The sign of the second order coeficient of Eq. (13) depends on $A_{1}$. Here we explain the condition $A_{1}>0$. We define the energy of the rest component relative to the true ground state energy, $E_{r}=\left\langle\psi_{r}|H| \psi_{r}\right\rangle-E_{0}$. The first moment $D_{1}$ is proportional to $E_{r}$, while the second moment $D_{2}$ is approximately proportional to $E_{r}^{2}$. Therefore their ratio $A$ has a $1 / E_{r}$ dependence. If $E_{r}$ increases as $t$ increases, $A$ decreases as a function of $t$, which means that $A_{1}>0$. In the following we consider two physically relevant but different truncation schemes that, however, share a common way for expanding shell model spaces as $t$ increases. The shell model orbits are divided into higher and lower orbits. As the parameter $t$ is the maximum nucleon number in the higher orbits, we expand truncated spaces by including basis states with larger spherical single particle energies on average. By this construnction of the series of truncation spaces, as $t$ increases, the rest component is described by basis states with larger spherical single particle energies and $E_{r}$ becomes larger. Therefore upwardly concave feature is generally expected in energy - energy variance plot.

Next we consider the expectation value of an observable $\hat{O}$, where a similar second order extrapolation formula holds. In the same way as the energy, we define the difference as

$$
\delta O=\langle\hat{O}\rangle-\langle\hat{O}\rangle_{0}
$$

that can be rewritten by 11]

$$
\delta O \sim d^{2} D_{1}^{O} E_{0},
$$

in which $D_{1}^{O}$ is defined as

$$
D_{1}^{O}=\sum_{n, m \neq 0} \frac{c_{n} c_{m}\left(O_{n m}-O_{00} \delta_{n m}\right)}{E_{0}},
$$

where $O_{n m}=\left\langle\psi_{m}|\hat{O}| \psi_{n}\right\rangle$. By eliminating $d^{2}, \delta O$ can be expanded by the ratio $\Delta E / E_{0}^{2}$ as

$$
\delta O=B \Delta E+\left(B A^{2}\right) \Delta E^{2}+\ldots,
$$

with

$$
B=\frac{D_{1}^{O}}{D_{2} E_{0}} .
$$

We assume that $B$ can be expanded as

$$
B=B_{0}+B_{1} \Delta E+\ldots
$$


where $B_{0}$ and $B_{1}$ are coefficients. Then, we obtain the second order formula for $\delta O$ as

$$
\delta O=B_{0} \Delta E+\left(B_{0} A_{0}^{2}+B_{1}\right) \Delta E^{2}+\ldots
$$

As a further large-scale numerical test, we investigate the ground state of ${ }^{64} \mathrm{Zn}$, whose $M$-scheme dimension is about 501 Million and its exact shell model calculation is quite difficult. However, exact results are reported in Ref. [15].

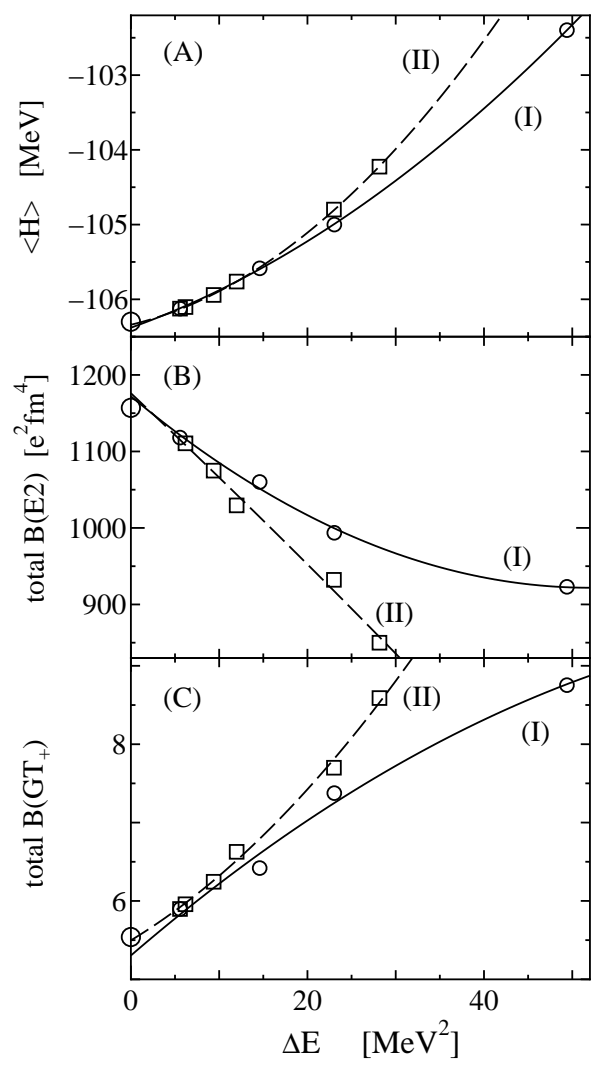

FIG. 3: (A) Second order extrapolations for the energy to the zero energy variance for the $0_{1}^{+}$state of ${ }^{64} \mathrm{Zn}$. (B, C) Extrapolations of the $B(E 2)(B)$ and Gamow-Teller (C) sum rules for the $0_{1}^{+}$state into zero energy variance. Exact results [15] are shown by open circles. The effective charges are taken as $e_{p}=1.35 e$ and $e_{n}=0.35 e$. For the oscillator length, $b=1.01 A^{1 / 6} \mathrm{fm}$ is taken. Unquenched Gamow-Teller operator are used. The solid and dashed lines show the results of truncation schemes (I) and (II), respectively.

In Fig. 3, the results of extrapolations of energies (A), $B(E 2)(B)$ and Gamow-Teller (C) sum rules for the $0_{1}^{+}$ state are shown for ${ }^{64} \mathrm{Zn}$. We consider two truncation schemes. For the truncation scheme (I), we plot the results for $t=1 \sim 4$ spaces, whose $M$-scheme dimension is $0.2 \sim 55$ Millions. For the truncation scheme (II), we plot the results for $t=4 \sim 8$ with $t_{0}=4$, whose $M$-scheme dimension is $0.2 \sim 40$ Millions. Shell model energies of truncated spaces can be well-fitted by the quadratic curves for both the truncation schemes. Extrapolated energies are -106.38 and $-106.34 \mathrm{MeV}$, for the truncation schemes (I) and (II), respectively. The exact ground state energy is $-106.3 \mathrm{MeV}$ [15]. The present extrapolated energies are quite close to the exact one. The extrapolated values of the total $\mathrm{B}(\mathrm{E} 2)$ are $1.17 \times 10^{3}$ and $1.18 \times 10^{3} e^{2} \mathrm{fm}^{4}$ for the truncation schemes (I) and (II), respectively, while the exact value is $1157 e^{2} \mathrm{fm}^{4}$ 15 with the effective charges as $e_{\pi}=1.35 e$ and $e_{\nu}=0.35 e$. Here we use the second order extrapolation formula for the expectation values. The extrapolated values of the total B(GT+) with unquenched Gamow-Teller operators are 5.3 and 5.5 for the truncation schemes (I) and (II), respectively, while its exact value is 5.54. The present extrapolations with two truncation schemes can give almost exact values.

In summary, we have proposed a second order extrapolation method for shell model calculations. Shell model energies of the truncated spaces as a function of $\Delta E$ are well described by a quadratic curve and the exact shell model energy is extrapolated in the limit $\Delta E \rightarrow 0$. To derive this second order extrapolation formula, it is quite important to consider the second order term of the $d^{2}$ expansion and the structure change of rest components in terms of the ratio of the moments $A$. By numerical solvable shell model calculations, we have compared the extrapolated energies to the exact one and showed that their accuracy is improved by the present second order extrapolation and that the first order extrapolation gives a lower limit of the shell model energy. In addition, we have extended the derivation of second order extrapolation to any observable. Finally, we have shown that different truncation schemes have different behavior in extrapolation, but, in principle, extrapolated values must be the same. This fact is used to confirm the accuracy of extrapolated values.

We thank Professor N. Yoshinaga and Dr. M. Serra for reading the manuscript and delightful comments. This work was supported from 'Research for the Future Program' by Japan Society for the Promotion of Science under grant RFTF97P01103, and was supported in part by Grant-in-Aid for Scientific Research (A)(2) (10304019), and one for Specially Promoted Research (13002001) from the Ministry of Education, Science and Culture.
[1] S. E. Koonin, D. J. Dean, and K. Langanke, Phys. Repts. 577, 1 (1996).

[2] J. Dukelsky, S. Pittel, Phys. Rev. C63 061303 (2001);
J. Dukelsky, S. Pittel, S. S. Dimitrova, M. V. Stoitsov, Phys. Rev. C65, 054319 (2002).

[3] A. Petrovici, Nucl. Phys. A704, 144c (2002). 
[4] M. Honma, T. Mizusaki and T. Otsuka, Phys. Rev. Lett. 75, 1284 (1995).

[5] T. Otsuka, M. Honma, T. Mizusaki, N. Shimizu, and Y. Utsuno, Prog. Part. Nucl. Phys. 47, 319 (2001).

[6] E. Caurier, F. Nowacki, A. P. Zuker, G. MartinezPinedo, A. Poves, and J. Retamosa, Nucl. Phys. A654, 747c (1999).

[7] M. Horoi, A. Volya and V. Zelevinsky, Phys. Rev. Lett. 82, 2064 (1999); M. Horoi, B. A. Brown and V. Zelevinsky, Phys. Rev. C65, 027303 (2002).

[8] T. Mizusaki and M. Imada, Phys. Rev. C65, 064319 (2002).

[9] M. Imada and T. Kashima, J. Phys. Soc. Jpn. 69,
$2723(2000)$.

[10] S. Sorella, Phys. Rev. B64, 024512 (2001).

[11] T. Kashima, M. Imada, J. Phys. Soc. Jpn. 70, 2287 (2001).

[12] For example see, T. Kashima and M. Imada, J. Phys. Soc. Jpn., 71, 3052 (2001); H. Morita, S. Watanabe and M. Imada, J. Phys. Soc. Jpn., 71, 2109 (2002), Y. Noda and M. Imada, Phys. Rev. Lett., 89, 176803 (2002).

[13] T. Mizusaki, RIKEN Accel. Prog. Rep. 33, 14 (2000).

[14] A. Poves and A. P. Zuker, Phys. Repts. 70, 235 (1981).

[15] E. Caurier, G. Martinez-Pinedo, F. Nowacki, A. Poves, J. Retamosa, A. P. Zuker, Phys. Rev. C59, 2033 (1999). 